\title{
The effects of familiarity in a perceptual matching task*
}

\author{
THOMAS P. FRIDEN \\ University of New Mexico, Albuquerque, New Mexico 87131
}

\begin{abstract}
Latencies of same-different judgments to pairs of two-digit numerals were recorded for stimuli presented in familiar or unfamiliar (inverted) orientation. Familiar stimuli were responded to more quickly than unfamiliar. For both stimulus types, latencies were correlated with the syllable length of the verbal representation of the numerals, allowing the interpretation that the effect of stimulus orientation is on encoding processes. In two other experiments, it was found that familiarity had no effect on different judgments when the stimuli were relatively simple (e.g., a single digit), but did affect different judgments with more complex stimuli. These results were related to the hypothesis that the complexity of verbal material determines whether different judgments are instigated by visual or by verbal representations of the stimuli.
\end{abstract}

The effects of familiarity on perception have recently been investigated in a class of research studies that have used as a dependent measure the latency of same-different judgments. Familiarity has been manipulated by presenting English letters, CVCs, or words either in an inverted orientation or with mirror reversal, and reaction time of same-different judgments to pairs of these stimuli is compared to reaction time to the same stimuli in their normal, upright positions. At least implicitly, this methodology is proposed as one that has several advantages over other methods of investigating familiarity. First, the simple manipulation of inverting stimuli is assumed to affect their familiarity alone, leaving other properties of the stimuli (e.g., complexity) constant between familiar and unfamiliar orientations. Second, the perceptual matching task may allow investigation of properties of perceptual processing uncontaminated by memorial factors, at least when the elements of each pair are presented in temporal and spatial proximity. That is, the task is a simple one for the $O$ and makes few, if any, demands of memory. Third, the responses required for familiar and unfamiliar stimuli are equated, allowing the assumption that stimulus availability is not confounded with response availability.

One important hypothesis concerning familiarity derived using this methodology comes from Hochberg (1968). Suggesting that familiarity can be viewed as either influencing what is seen in a momentary glance, or as influencing the efficiency of remembering what is seen, Hochberg presents data that are consistent with the latter position. In particular, when the elements of a pair of stimuli were presented with no temporal delay and only slight spatial separation, reaction times were equal for familiar stimuli (English words) and unfamiliar

*This research is based, in part, on a paper presented at the Midwestern Psychological Association Convention in Cleveland, Ohio, 1972. It was supported in part by the University of New Mexico Research Allocations Board. I wish to thank Cathy Bennett and Lorenzo Espinisa for assistance in data collection and analysis. Requests for reprints should be sent to Thomas Friden, Department of Psychology, University of New Mexico, Albuquerque, New Mexico 87131. stimuli (mirror reversals of English words). However, with either a temporal delay or a large spatial separation (20 deg) between the elements of each pair, latencies of responses to the familiar stimuli were faster than to the unfamiliar. Presumably, manipulation of either spatial separation or temporal delay increases the O's memorial burden. Familiar stimuli are more efficiently remembered than unfamiliar stimuli, producing the obtained effect.

Egeth and Blecker (1971), in a series of experiments using single letters and CVCs, have reported data at variance with that of Hochberg. Presenting these stimulus materials with no temporal delay and only slight spatial separation, inverted stimuli were responded to less quickly than upright stimuli when the elements of the pair were the same. No effect of familiarity was found for stimuli having different elements. There are many procedural differences between Hochberg's and Egeth and Blecker's experiments that may have resulted in the discrepancy, and these are discussed by Egeth and Blecker.

Of more immediate interest is the status of Hochberg's hypothesis that familiarity influences the efficiency of remembering stimuli. This hypothesis is still viable if one assumption about the perceptual matching task is questioned. This is that it makes little requirement of memory. Two lines of evidence, obtained with familiar stimuli, suggest that, under some conditions, this assumption is false. The first is that the effect of familiarity may not be due to familiarity per se, but rather to pronounceability, at least with some verbal materials (Egeth \& Blecker, 1971; Gibson, Bishop, Schiff, \& Smith, 1964). Egeth and Blecker found that if two stimuli were roughly equated in familiarity but differed in pronounceability (e.g., FBI and FIB), the more easily pronounceable was responded to more quickly, i.e., same judgments were faster. A second bit of evidence comes from Klapp (1971), who found that latencies of same-different judgments to two-digit numerals were correlated with the syllable length of the name of the numerals. Taken together, this evidence is consistent with the suggestion that even when elements of a pair of stimuli are presented with neither temporal 
Table 1

Average Latencies in Milliseconds from Experiment I

\begin{tabular}{|c|c|c|c|c|c|c|c|c|}
\hline \multirow{3}{*}{$\begin{array}{l}\text { Orientation } \\
\text { Familiar }\end{array}$} & \multirow{3}{*}{$\begin{array}{l}\begin{array}{l}\text { Judg- } \\
\text { ment }\end{array} \\
\text { Same } \\
\text { Diff } \\
\text { Mean }\end{array}$} & \multicolumn{6}{|c|}{ Syllable Length } & \multirow{3}{*}{$\begin{array}{c}\text { Mean } \\
535 \\
581 \\
558\end{array}$} \\
\hline & & \multicolumn{2}{|c|}{2} & \multicolumn{2}{|c|}{3} & \multicolumn{2}{|c|}{4} & \\
\hline & & $\begin{array}{l}515 \\
576 \\
546\end{array}$ & $\begin{array}{l}(2.3) \\
(4.6)\end{array}$ & $\begin{array}{l}542 \\
584 \\
563\end{array}$ & $\begin{array}{l}(2.7) \\
(2.3)\end{array}$ & $\begin{array}{l}549 \\
583 \\
566\end{array}$ & $\begin{array}{l}\text { (3.3) } \\
\text { (3.1) }\end{array}$ & \\
\hline Unfamiliar & $\begin{array}{l}\text { Same } \\
\text { Diff } \\
\text { Mean }\end{array}$ & $\begin{array}{l}550 \\
598 \\
574\end{array}$ & $\begin{array}{l}(1.5) \\
(3.8)\end{array}$ & $\begin{array}{l}626 \\
624 \\
625 \\
\end{array}$ & $\begin{array}{l}(5.3) \\
(5.8)\end{array}$ & $\begin{array}{l}605 \\
642 \\
624 \\
\end{array}$ & $\begin{array}{l}(6.0) \\
(2.1)\end{array}$ & $\begin{array}{l}594 \\
621 \\
608\end{array}$ \\
\hline
\end{tabular}

Note-Percent errors are in parentheses.

delay nor large spatial separation, the Os may still respond on the basis of a verbal encoding of the stimuli. The duration of this encoding process is roughly correlated with syllable length necessary to pronounce the stimuli.

The implication of these data is clear. If Os make decisions in a same-different task on the basis of a verbal representation of the stimuli, then the possibility exists that Hochberg's hypothesis is correct. That is, familiarity influences the speed of this memorial process, and does not influence what is seen in a momentary glance. The first step in supporting this analysis is to demonstrate that unfamiliar stimuli are verbally encoded in a perceptual matching task. Experiment I attempts to do this by recording latencies of same-different judgments to two-digit numerals, either in familiar or unfamiliar orientations. Following Klapp (1971), if reaction time is correlated with the number of syllables necessary to pronounce these stimuli, evidence for same-difference decisions being based not on the visual forms but rather on verbal representations is obtained.

\section{EXPERIMENT I}

\section{Method}

\section{Subjects}

Three graduate and seven undergraduate students (four males) participated in six experimental sessions each, and were paid $\$ 6$ for their services.

\section{Apparatus and Stimuli}

The stimuli consisted of pairs of two-digit numerals, typed (pica) horizontally in black ink on white cards. The elements of each pair subtended a visual angle of $12 \mathrm{~min}$ high $\mathrm{x} 16 \mathrm{~min}$ wide, and the entire horizontal extent of both elements was $1 \mathrm{deg}$. Six of the two-digit numerals took two syllables to pronounce (14, $15,20,30,40$, and 50$)$, six took three syllables $(17,42,52,62$, 70 , and 95$)$, and six took four syllables $(37,47,57,76,78$, and 87). These were taken from Eriksen, Pollack, and Montague (1970), and appear to be representative of their respective classes. These stimuli were combined with a corresponding mate of the same syllable length to make 18 pairs of same stimuli and 18 pairs of different stimuli. Since all four-syllable two-digit numerals contain seven, two pairs were constructed with matching numerals in one corresponding position (e.g., 37-47) in each of the syliable-length sets. Two identical sets of 36 cards were prepared, one appearing in familiar orientation and the other in unfamiliar orientation, i.e., rotated $180 \mathrm{deg}$.

A Scientific Prototype two-channel tachistoscope, Model $800-\mathrm{F}$, was used to present the stimuli at a viewing distance of $84 \mathrm{~cm}$. Latency was measured as the time between stimulus onset and depression of one of two microswitches, labeled "same" and "different," and recorded by a Hunter Klockounter, Model 120A.

\section{Procedure}

The Os were instructed to respond as quickly as possible without making errors, and to avoid naming the stimuli by making the same-different judgment a visual one. They were told in advance the number of trials per session, and were given as many practice trials as they felt were necessary until they were comfortable in dealing with the response keys. Half of the Os pressed the "same" key with the thumb of their preferred hands and the "different" key with the thumb of their other hands. This was reversed for the other half of the Os.

There were 144 trials in each session, during which the mixed list of familiar and unfamiliar stimuli were presented in shuffled order. Each trial consisted of a 1-sec presentation of a single pair of numerals, followed by a 6-sec intertrial interval, during which time a small fixation cross was present in the tachistoscope.

The first two sessions were considered practice, and the data from these were not analyzed. Because of a slight error in positioning the numerals on the card, the data from one stimulus (62-62 in unfamiliar orientation) were not included in the analysis.

\section{Results and Discussion}

On $3.5 \%$ of the trials, an incorrect response was given, and these data are omitted from the following analyses. The error rate is low enough for the conclusions reached probably to be unaffected by eliminating these data (Egeth \& Smith, 1967).

The median latency for each $\mathrm{O}$ across sessions was computed for the 12 experimental conditions, and the means of these are presented in Table 1. A three-factor analysis of variance was performed on these data, Judgment by Familiarity by Syllable Length, and all three main effects are significant. Same judgments were faster than different judgments, $F(1,9)=14.80$, $p<.01$; familiar stimuli were responded to more quickly than unfamiliar stimuli, $F(1,9)=17.58, p<.01$; and there were differences as a function of the number of syllables necessary to pronounce the stimuli, $F(2,18)=13.04$, $p<.001$. The general trend of this variable is that of increasing latency as syllable length increases, although 
by no means is this a perfect relationship. The Syllable Length by Judgment interaction is significant, $F(2,18)=$ $6.42, p<.01$, and is due to the effects of syllable length being monotonic with different judgments, but not with same judgments. There was a nonsignificant reversal in means for same judgments between the three $(M=$ $584 \mathrm{msec})$ and four $(M=577 \mathrm{msec})$ syllable length stimuli $[\mathrm{t}(9)=.79]$.

The effects of syllable length on reaction time are small in terms of mean differences, but are consistent with other studies that have attempted to assess the rate of implicit speech. The linear least-squares function relating the syllable length of the numerals to prediction of overall mean reaction time (column means in Table 1) has a slope of $8 \mathrm{msec}$ per syllable, presuming that both elements in a stimulus pair are verbally encoded. This is of an order of magnitude similar to that found by Eriksen et al (1970) of $11 \mathrm{msec}$ per syllable and by Klapp (1971) of $8.5 \mathrm{msec}$ per syllable.

Thus, the main results of this experiment support the notions that both familiar and unfamiliar stimuli were being verbally encoded; that the length of this transfer from iconic to short-term storage was correlated with the syllables necessary to pronounce the stimuli; and that same-different decisions, at least on some trials, were made on the basis of the verbal representation of the stimuli, rather than their visual images. Further, these data invite, but do not demand, speculation that the effects of familiarity in a perceptual matching task are on encoding processes. That is, consistent with the data is the suggestion that the transfer from iconic storage to short-term memory is influenced by inversion of stimuli.

Further analysis of these data indicate that these conclusions are also valid after considerable practice. Analyzing the data from the sixth session only, prior to which each $O$ had received 720 trials with these stimuli, all main effects are still found to be significant: for judgment, $F(1,9)=6.76, p<.05$; for familiarity, $F(1,9)$ $=10.66, p<.01$; and for syllable length, $F(2,18)=6.33$, $p<.01$. No significant interactions are present.

There is one puzzling aspect of these data, and this concerns the effect of familiarity on different judgments. Egeth and Blecker (1971), using either pairs of letters or CVC trigrams, found that stimuli presented in unfamiliar orientation were responded to more slowly only when the elements of the pair were identical. For different judgments, no effects of familiarity were evidenced. In the present experiment, familiarity influenced both same and different judgments, indicated by the significant main effect of familiarity, coupled with the absence of a significant Judgment by Familiarity interaction, $F(1,9)=1.69, p>.20$. Further, a test for the simple effect of familiarity on different judgments yields $t(9)=3.69, p<.01$. Experiment II was performed on the same practiced Os, after all data in the main experiment had been collected, using pairs of letters as stimuli. The purpose was an attempt to
Table 2

Average Latencies in Milliseconds from Experiment 1

\begin{tabular}{lrrrr} 
& \multicolumn{3}{c}{ Judgment } \\
\cline { 2 - 5 } Orientation & \multicolumn{2}{c}{ Same } & \multicolumn{2}{c}{ Different } \\
\hline Familiar & 506 & $(2.8)$ & 543 & $(1.7)$ \\
Unfamiliar & 545 & $(15.6)$ & 541 & $(2.5)$ \\
Difference & -39 & & 2 & \\
\hline
\end{tabular}

Note-Percent errors are in parentheses.

replicate Egeth and Blecker's findings concerning the effects of familiarity on different judgments in the present experimental situation, and in particular with experienced Os.

\section{EXPERIMENT II}

\section{Method}

\section{Subjects}

The same 10 Os who participated in Experiment I were tested in two additional sessions, and were paid $\$ 2$ for their services.

\section{Apparatus, Stimuli, and Procedure}

The apparatus was identical to that used in Experiment I.

Stimulus cards were prepared as in Experiment I, except that the elements of each pair were single letters. Letters which were similar in upright and inverted orientation were not used. Random mates were selected to form the different pairs. Two identical decks of 36 cards were made, with one rotated 180 deg for the unfamiliar orientation. The elements of each pair subtended a visual angle of $12 \mathrm{~min}$ high $\times 8 \mathrm{~min}$ wide, and the entire horizontal extent of both elements was $51 \mathrm{~min}$.

The procedure, except for appropriate instructional changes, was identical to that of Experiment $I$. The mixed list of familiar and unfamiliar stimuli was presented in shuffled order for a total of 144 stimulus presentations during each session. The first session was considered practice, and data from it are not included here.

\section{Results and Discussion}

The median latency for each O's correct responses on the second session was computed for the four experimental conditions, and the means of these are presented in Table 2. A two-factor analysis of variance, Judgment by Familiarity, performed on these data reveals that neither main effect is significant, although the effect of familiarity approaches significance, $F(1,9)=4.55$. $\mathrm{p}<.10$. The Judgment by Familiarity interaction is significant, $F(1,9)=12.72, p<.01$. Presence of this interaction is due to familiarity influencing same judgments, $t(9)=-2.95, p<.05$, but not different judgments, $t(9)=.39$, n.s.

Error rates are also included in Table 2, and are sufficiently low for three cells to indicate that the conclusions reached are probably unaffected by eliminating these data. Although the error rate of $15.6 \%$ for same judgments of the unfamiliar stimuli is high enough to affect that cell mean, it does not compromise interpretation of the effects of familiarity within 
Table 3

Average Latencies in Milliseconds from Experiment III

\begin{tabular}{lcccccc}
\hline & \multicolumn{8}{c}{ Two Digits } \\
\cline { 3 - 7 } Orientation & Single Digit & \multicolumn{3}{c}{ One Diff } & Two Diff \\
\hline Familiar & 543 & $(3.0)$ & 737 & $(3.0)$ & 666 & $(2.5)$ \\
Unfamiliar & 574 & $(3.5)$ & 966 & $(10)$. & 960 & $(5.5)$ \\
Difference & -31 & \multicolumn{7}{c}{ Same Judgment } \\
& \multicolumn{7}{c}{ Different Judgment } & -229 & & -294 & \\
Familiar & 595 & $(3.5)$ & 820 & $(5.5)$ & 812 & $(2.5)$ \\
Unfamiliar & 590 & $(3.0)$ & 960 & $(4.5)$ & 908 & $(6.0)$ \\
Difference & 5 & \multicolumn{7}{c}{-140} & & -96 & \\
\hline
\end{tabular}

Note-Percent errors are in parentheses.

response types. This is not a speed-accuracy tradeoff, because the two measures of performance, latencies and error, are positively correlated for same judgments, indicating that each is reflective of the same effect, i.e., the processing demands of judging identical stimuli in unfamiliar orientation.

This experiment is a qualitative replication of Egeth and Blecker (1971) and supports the finding that, with simple verbal stimuli, familiarity affects only same judgments. Further, it extends their results by demonstrating that stimulus orientation is an effective variable after considerable practice. Comparing this experiment to Experiment I seems to implicate complexity, defined as the number of verbal stimuli making up the elements of each pair, as the variable controlling whether orientation will influence difference judgments.

This supposition was tested in a third experiment by varying the number of digits making up the elements of each pair. A second variable was also manipulated, one that may determine whether inversion will affect difference judgments. This concerns how many of the digits making up the elements of each different pair are, in fact, different (Eichelman, 1970). If single digits are used as elements, all digits differ. When two or more digits are used as elements, there are several ways of constructing pairs of different stimuli, and these may not be equivalent in terms of the processing demands placed on the Os. If only one digit of those composing one element is different from the digits composing the other element, there may be considerable difficulty in making difference judgments on the basis of the visual images because of the identity of part of those images from each element. A more efficient strategy may involve decisions based on verbal representations, in which case stimulus orientation should be an effective variable, according to Hochberg (1968).

In Experiment I, the different stimulus pairs were constructed in both fashions, i.e., one digit different on one-third of the trials and both digits different on the remainder, because of the constraints produced by the limited number of stimuli. Experiment III was designed to manipulate this variable systematically in order to test the hypothesis that inversion would increase the latencies of different judgments only when the visual images were relatively confusable.

\section{EXPERIMENT III}

\section{Method}

\section{Subjects}

The Os were 30 undergraduates (15 males), who received bonus points in an undergraduate course for participation.

\section{Apparatus, Stimuli, and Procedure}

The apparatus was identical to that used in Experiment I.

Three sets of stimulus cards were prepared, using the digits 2 , $3,4,5$, and 7 . The 10 Os in Group 1 received 80 trials, using stimuli whose elements were single digits. Random mates were used to form the 40 different stimuli. The 10 Os in Group 2 received 80 trials with stimuli whose elements consisted of two-digit numerals. The 40 stimuli for different judgments were constructed so that only one of the digits differed between the elements of each pair. The $10 \mathrm{Os}$ in Group 3 also received 80 trials with pairs of two-digit numerals, but the 40 stimuli for different judgments were constructed so that both of the digits differed between the elements of each pair. This was accomplished by re-pairing the identical stimuli used for Group 2. For all of the groups, two identical decks of 40 stimuli were prepared, and one of these was presented in inverted orientation to form the unfamiliar stimuli. Stimuli for Groups 2 and 3 were prepared as in Experiment I; stimuli for Group 1 were prepared as in Experiment II.

The procedure, except for appropriate instructional changes, was identical to Experiment I. Six practice trials were given at the beginning of the session, but are not included in the data analysis.

\section{Results and Discussion}

The overall error rate was $4.6 \%$, and these trials were excluded from analysis. The median latency of correct judgment for each $O$ was computed for the 12 experimental conditions, and the means of these are contained in Table 3.

\section{Same Judgments}

A two-factor, Groups by Familiarity, analysis of variance performed on the latencies of same judgments revealed main effects of group, $F(2,27)=14.04$, $\mathrm{p}<.001$, with two-digit stimuli having longer latencies, and familiarity, $F(1,27)=106.09, p<.001$, with inverted stimuli having longer latencies than upright stimuli. The Group by Familiarity interaction was also highly significant, $F(2,27)=19.47, p<.001$. Testing for the simple effects of familiarity within groups reveals that for all three groups, familiar stimuli were responded to more quickly than were unfamiliar stimuli: for single digits (Group 1), $\mathrm{t}(9)=2.86, \mathrm{p}<.05$; for two digits, one different (Group 2), $t(9)=5.63, p<.001$; and for two digits, both different (Group 3), $t(9)=8.78, p<.001$. The interaction appears to be due to familiarity having 
less effect with single digits than with two digits. This hypothesis was tested by an application of Duncan's multiple range test to the three groups, using as scores the difference in response latency to familiar and unfamiliar stimuli. At the 05 confidence level, both groups receiving two digits as elements of each pair showed a larger effect of familiarity than the group receiving a single digit as the element of each pair. The former groups did not show differences, however, supporting the hypothesis that familiarity is relatively more effective on same judgments when the stimuli are complex.

\section{Different Judgments}

A similar two-factor analysis of vatiance performed on latencies of different judgments reveals that the two-digit stimuli took longer response times, $F(2,27)=$ $14.94, \mathrm{p}<.001$, and that over all groups, familiar stimuli were responded to more quickly than unfamiliar, $F(1,27)=29.21, p<.001$. The latter statement is qualified by the presence of a Group by Familiarity interaction, $F(2.27)=9.11, p<.001$. Tests for simple effects indicate that this interaction is due to a nonsignificant reversal of the effects of stimulus orientation for the group receiving single digits as elements, $t(9)=-.47$, and significant effects of familiarity for the groups receiving two digits as elements, $\mathrm{t}(9)=4.84, \mathrm{p}<.001$ (Group 2), and $\mathrm{t}(9)=$ $3.27, p<.01$ (Group 3). For these latter two groups, the effect of orientation when only a single digit differed (140 msec, Group 2) was slightly larger than when both digits differed (96 msec, Group 3), although this difference fails to reach statistical significance, $t(18)=$ 1.06 .

These results qualitatively replicate Experiments I and II with naive Os, and further implicate the role of complexity in determining whether different judgments are slowed in response to inverted stimuli. They rule out the unlikely hypothesis that single digits as stimuli are processed in a different fashion than single letters, in that the pattern of results for Group 1 is similar to that of Experiment II. That is, familiarity influences same, but not different, judgments with simple, verbal stimuli.

With more complex stimuli, stimulus orientation is effective, even when the visual images are relatively unconfusable, i.e., when all elements of the stimuli are different. The number of digits that differ in the elements of each pair is clearly not a powerful variable in controlling the effects of familiarity, at least with the limited range of this variable and the naive Os used in this study. Other studies have indicated that it is an important variable in determining speed of same-different responses (Bamber, 1969, 1972; Eichelman, 1970), but further research is needed to implicate its role in relation to stimulus orientation.

\section{GENERAL DISCUSSION}

Considering the data obtained in Experiment $I$, one assumption about the nature of the perceptual matching task is clearly not supported. This assumption is that the simple task of the $O$ may allow investigation of properties of perceptual processing uncontaminated by memorial factors. The obtained relationship between syllable length of the numerals and latencies of judgment for both familiar and unfamiliar stimuli contraindicates this assumption, in that, at least on some of the trials, the Os were making same-different judgments on the basis of the verbal representation of the numerals rather than their visual images.

In addition, the pattern of results for the three experiments is consistent with the suggestion that the nature of the effect of stimulus orientation is on encoding processes, given that complexity determines whether different responses will be instigated by verbal or by visual representations of the stimuli. Egeth and Epstein (1972), using considerably different experimental procedures, have argued that different judgments to simple verbal stimuli (i.e., pairs of single letters) are based on a visual-spatial match of the elements; Experiment I indicates that more complex different elements are verbally encoded. The present three experiments demonstrate an effect of orientation only in the latter case, and assuming no effects of familiarity for a visual-spatial match describes the equivalence, with respect to orientation, of the different judgments to single-element stimuli. Egeth and Epstein (1972) also argue that same judgments are based on verbal representations of single-element verbal stimuli; Experiment $I$ indicates that more complex stimuli with identical elements are also verbally encoded. Thus, Hochberg's hypothesis (1968), that the effect of stimulus orientation is on memorial processes, is predictive of the obtained effect of stimulus orientation on same judgments regardless of the complexity of the elements, and the obtained effect of stimulus orientation on different judgments when the stimuli are complex and verbally encoded, and the lack of effect when the stimuli are simple and different judgments are based on visual matches.

The data invite such speculation, but do not demand the interpretation that stimulus orientation influences encoding processes directly. It is possible that familiarity has its effects prior to when the stimuli are verbally represented. For example, the increased latency of response to inverted stimuli could be relegated to the time necessary for mental rotation to an upright position (e.g., Shepard \& Metzler, 1971), after which process the verbal representation is formed. Further, it is clear that a model implying that all variance in same-different judgments is due to verbal representations is too simple. In Experiment $I$, there was an interaction between type of judgment and syllable length, and the relationship of 
latency and syllable length was not monotonic for all familiarity-judgment types of stimulus combinations. Thus, any detailed description of performance must necessarily be more complex than a simple description of the time course of verbal encoding.

These conclusions may be qualified further by the methodology used in the present series of studies, ${ }^{1}$ in which the Os were required to process stimuli in inverted orientation as part of a larger set of stimuli, some of which appeared in upright position. It is possible that the presence of these normal verbal stimuli encouraged a general verbal-processing set, producing similar processing of the inverted stimuli. Thus, a design in which stimulus orientation was a between-Ss variable could eliminate verbal encoding of the unfamiliar stimuli.

\section{REFERENCES}

Bamber, D. Reaction times and error rates for "same"-"ddifferent" judgments of multidimensional stimuli. Perception \& Psychophysics, 1969, 6, 169-174.

Bamber, D. Reaction times and error rates for judging nominal identity of letter strings. Perception \& Psychophysics, 1972, $12,321-326$.

Egeth, H., \& Blecker, D. Differential effects of familiarity on judgments of sameness and difference. Perception \& Psychophysics, 1971, 9, 321-327.
Egeth, H., \& Epstein, J. Differential specialization of the cerebral hemispheres for the perception of sameness and difference. Perception \& Psychophysics, 1972, 12, 218-220.

Egeth, H. E., \& Smith, E. E. On the nature of errors in a choice reaction time task. Psychonomic Science, 1967, 8, 345-346.

Eichelman, W. H. Familiarity effects in the simultaneous matching task. Journal of Experimental Psychology, 1970, 86, 275-282.

Eriksen, C. W., Pollack, M. D., \& Montague, W. E. Implicit speech: Mechanism in perceptual encoding? Journal of Experimental Psychology, 1970, 84, 502-507.

Gibson, E. J., Bishop, C. H., Schiff, W., \& Smith, J. Comparison of meaningfulness and pronunciability as grouping principles in the perception and retention of verbal material. Journal of Experimental Psychology, 1964, 67, 173-182.

Hochberg, J. In the mind's eye. In R. N. Haber (Ed.), Contemporary theory and research in visual perception. New York: Holt, Rinehart \& Winston, 1968.

Klapp, S. T. Implicit speech inferred from response latencies in same-different decisions. Journal of Experimental Psychology, $1971,91,262-268$.

Shepard, R. N., \& Metzler, J. Mental rotation of three-dimensional objects. Science, 1971, 171, 701-703.

\section{NOTE}

1. A thoughtful reviewer pointed out this possibility.

(Received for publication April 25, 1973; revision received June 22,1973 .) 